\title{
Deformation Modelling Based on PLSR for Cardiac Magnetic Resonance Perfusion Imaging
}

\author{
Jianxin Gao ${ }^{1}$, Nick Ablitt ${ }^{1}$, Andrew Elkington ${ }^{2}$, and Guang-Zhong Yang ${ }^{1,2}$ \\ ${ }^{1}$ Royal Society/Wolfson Foundation MIC Laboratory, Imperial College, London, UK \\ ${ }^{2}$ Royal Brompton Hospital, London, UK \\ \{jxg, naa99, gzy@doc.ic.ac.uk\}
}

\begin{abstract}
This paper introduces a novel approach to deformation modelling based on partial-least-squares-regression and its application to the registration of first-pass magnetic resonance perfusion image sequences. The method relies on the extraction of intrinsic correlations between the latent factors of both the input and output signals for deriving a simple yet accurate deformation model. The strength of the technique has been demonstrated with both numerically simulated data sets and a myocardial perfusion study which involves nine patients with known coronary artery disease. The method represents a step forward for the commonly used principal component analysis for salient motion feature extraction. The proposed technique should be applicable to studies that involve deformation prediction such as motion adaptive radiotherapy and imaging, where the deformation of the target organ can be predicted from externally measurable signals.
\end{abstract}

\section{Introduction}

Magnetic Resonance (MR) myocardial perfusion imaging is a relatively new technique for distinguishing ischemic myocardium from healthy tissue [1,2]. It offers the possibility of non-invasively determining the location and extent of ischemia or infarction at transmural resolutions. First-pass techniques using fast gradient echo (turboFLASH) or echo-planar (EPI) sequences are now common practice in clinical research and quantitative results have been achieved in animal studies with intravascular agents (polylysine-Gd-DTPA) as a macromolecular blood pool marker [3-5]. At the same time, semi-quantitative results have also been established in humans with conventional extracellular agents (Gd-DTPA). Either approach has an impact on the detailed characterisation of the relationship between functional and perfusion abnormalities.

For first-pass perfusion imaging, a complete 2D or volumetric data set has to be acquired for each cardiac cycle [6-8]. Cardiac and respiratory induced motion is a major problem during both imaging and subsequent perfusion analysis. With standard perfusion sequences, a typical single slice short axis image of the myocardium may take about $100 \mathrm{~ms}$ to acquire. Even with limited coverage of three to four slices of the left ventricle, the acquisition window within each cardiac cycle can still become excessive. During this period, cardiac motion can cause the myocardium imaged through different image planes to be mis-registered, i.e., some parts of the myocar- 
dium may be imaged multiple times whereas other parts may be missed out completely. This type of mis-registration is difficult to correct for by using postprocessing techniques. In a previous study, we have proposed a novel 4D motiondecoupling technique based on the use of motion tagging and slice tracking [9]. This has simplified the problem to multiple $2 \mathrm{D}$ temporal free-form image registration tasks. In this case, the deformation within the imaging plane is normally caused by respiration during the acquisition period, typically lasting for about 50 cardiac cycles. Breath-holding has been suggested, but for most patients, particularly those with coronary artery disease, this has proven to be impractical. This renders accurate image registration of cardiac deformation a crucial step in the quantification of perfusion indices. Thus far, several image registration approaches have been proposed for inplane motion correction $[10,11]$. The purpose of this paper is to introduce the concept of partial-least-squares-regression (PLSR) [12] to derive intrinsic characteristics of the deformation model both for improving the internal consistency of the registration process and for predicting myocardial deformation due to respiration.

\section{Material and Methods}

\subsection{Myocardial Motion Prediction}

Existing research in cardiac imaging has shown that there is significant correlation between myocardial deformation and respiratory motion patterns. In MR coronary imaging, for example, respiratory navigators have been extensively used to sample the movement of the diaphragm in order to predict the distortion of the epicardial surface, thus allowing real-time adaptive tracking of the coronary arteries to be used to avoid breath-holding for patient studies. Research has also shown that the internal correlation between respiration and cardiac deformation is subject specific, but intra-patient characteristics remain relatively consistent throughout the imaging period. Inspired by these findings, we propose to use the varying intensity distribution of the chest and diaphragm as predictors of myocardial deformation.

Historically, models for respiratory induced cardiac motion have been based on cardiac landmarks. This has the advantage of simplifying the derivation of motion models but has the major drawbacks in accuracy. Respiratory motion and its induced cardiac deformation are highly complex. In our proposed motion prediction model, principal modes of intensity variation related to the diaphragm and chest are extracted to correlate with deformation vectors of the myocardium, leading to a reliable motion prediction approach. Figure 1 illustrates the basic concepts involved in the motion prediction model. The use of free-form image registration allows the extraction of intra-frame tissue deformation, resulting in a dense displacement vector field within the image plane. To relate motion fields reflecting respiration to those of cardiac deformation, non-linear correlation techniques need to be employed. This, however, is not trivial especially when the model parameters are unknown and patient specific. By observing the fact that there is a large amount of redundancies and internal correlations in intensity distribution of the chest wall and the motion vectors of the myocardium, deformation modelling based on PLSR can be applied.

In PLSR, principal component analysis (PCA) is first applied to extract the intrinsic patterns, i.e. latent factors, of the original data sets. Then the focus is put on these latent factors rather than the original data that carries redundancies. A relationship can 
subsequently be established between these latent factors through linear regression. In practice, this is implemented as a learning process, i.e., by feeding the model with both input (intensity distributions around the chest wall and diaphragm) and observed output (motion vectors of myocardial deformation). Since intra-patient motion characteristics are relatively consistent throughout the imaging period, a short segment of the image sequence can be used for extracting the deformation model such that cardiac deformation can be automatically predicted from the intensity variations at the chest wall or the diaphragm. This removes the need of using image registration for the remaining images within the sequence. Of course, the same process can also be used for processing the entire sequence during learning so as to derive an internally consistent motion model to regulate the deformation vectors calculated from the registration algorithm.

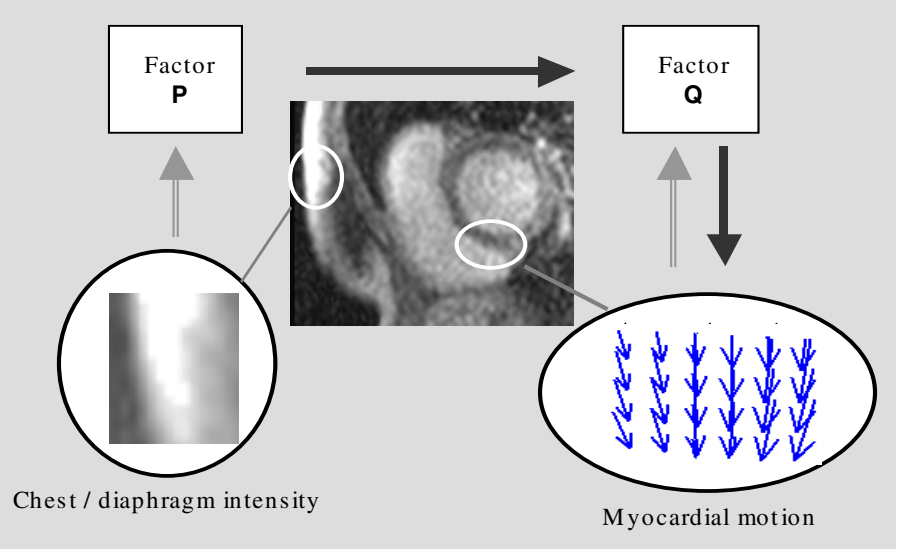

Fig.1. A schematic illustration of using PLSR for deformation modelling. In this example, multiple measurements of the intensity distribution near the chest wall, reordered as long vectors, are used as the input, from which input latent factors are extracted. A similar process is also applied to the observed output data, in this case the deformation vectors of the heart derived from normal free-form image registration. A model that reflects the intrinsic correlation between input/output can then be derived from these factors. At the prediction stage, the path along the dark arrows is followed, i.e., myocardial deformation can be directly predicted from the intensity pattern measured at the chest wall

\subsection{PLSR Implementation}

Let $\mathbf{X}$ denote the intensity pattern of the chest wall and/or the diaphragm, $\mathbf{Y}$ the freeform deformation vectors of the myocardium, each with a dimensionality of $p$ and $m$. When $n$ image frames are used for learning, their intrinsic relationship can be expressed as

$$
\mathbf{Y}=\mathbf{X C}+\mathbf{E}
$$

where $\mathbf{C}$ is a $p$ by $m$ coefficient matrix, $\mathbf{E}$ represents noise and higher order terms that are not accounted for in the above relationship, with the same rank of Y. In PLSR, both the input $\mathbf{X}$ and output $\mathbf{Y}$ are used for extracting the factors, also called scores, in forming the coefficient matrix $\mathbf{C}$. The regression is implemented based on these fac- 
tors rather than the original input and output matrix themselves. That is, PCA is applied to decompose $\mathbf{X}$ and $\mathbf{Y}$ as

$$
\begin{aligned}
& \mathbf{X}=\mathbf{T P}+\mathbf{E}_{1} \\
& \mathbf{Y}=\mathbf{T Q}+\mathbf{E}_{2}
\end{aligned}
$$

In Equation (2) and (3), $\mathbf{T}$ is the factor score matrix of $n$ by $c, \mathbf{P}$ the factor loading matrix of $c$ by $p, \mathbf{Q}$ the coefficient loading matrix of $c$ by $m$, here $c$ is the number of predominant latent vectors kept in the PCA computation; and $\mathbf{E}_{\mathbf{1}}$ and $\mathbf{E}_{\mathbf{2}}$ represent, respectively, parts of $\mathbf{X}$ and $\mathbf{Y}$ that are unaccounted for, with the same ranks as of $\mathbf{X}$ and $\mathbf{Y}$.

The latent vectors are the eigenvectors of the covariance matrix $\left(\mathbf{X}^{\prime} \mathbf{Y}\right)^{\prime}\left(\mathbf{X}^{\prime} \mathbf{Y}\right)$, through which a weighting matrix $\mathbf{W}$ can be computed iteratively such that $\mathbf{T}=\mathbf{X W}$. This means that the factor score matrix $\mathbf{T}$ is the weighting result of input matrix $\mathbf{X}$. The weighting matrix $\mathbf{W}$ is generated in such a way, that each of its columns will maximize the covariance between the response matrix and the corresponding factor scores. By applying a normal regression procedure, matrix $\mathbf{Q}$ can be derived which describes the relationship between $\mathbf{Y}$ and $\mathbf{T}$. For a predefined number of principal components $c$, usually much less than the number of predictors, the iteration procedure, known as the NIPALS algorithm $[13,14]$, is as follows:

\section{Initialise $\boldsymbol{A}=\boldsymbol{X}^{\prime} \boldsymbol{Y}, \boldsymbol{M}=\boldsymbol{X}^{\prime} \boldsymbol{X}, \boldsymbol{B}=\boldsymbol{I}$;}

2. For $k=1$ to $c$, begin iteration:

3. Compute coefficient loading vector $\boldsymbol{q}_{k}$, being the first eigenvector of $\boldsymbol{A}^{\prime} \boldsymbol{A}$;

4. Compute weighting (column) vector $\boldsymbol{w}_{k}=\boldsymbol{B} \boldsymbol{A} \boldsymbol{q}_{k}$, normalize $\boldsymbol{w}_{\boldsymbol{k}}=\boldsymbol{w}_{k} /\left\|\boldsymbol{w}_{k}\right\|$;

5. Compute factor loading vector (column) $\boldsymbol{p}_{k}=\boldsymbol{M} \boldsymbol{w}_{k} / f$, where $f=\boldsymbol{w}_{k}{ }^{\prime} \boldsymbol{M} \boldsymbol{w}_{k}$;

6. Renew the coefficient loading vector $\boldsymbol{q}_{k}$ as $\boldsymbol{q}_{k}=\boldsymbol{A}$ ' $\boldsymbol{w}_{k} / f$;

7. Renewal for $\boldsymbol{A}$ and $\boldsymbol{M}: \boldsymbol{A}=\boldsymbol{A}-f \boldsymbol{p}_{k} \boldsymbol{q}_{k}, \boldsymbol{M}=\boldsymbol{M}-f \boldsymbol{p}_{k} \boldsymbol{p}_{k}$;

8. Renewal for $\boldsymbol{B}: \boldsymbol{B}=\boldsymbol{B}-\boldsymbol{w}_{k} \boldsymbol{p}_{k}{ }^{\prime}$;

9. If $k>c$ or $M$ vanishes, stop iteration; otherwise return to step 3 .

where $\mathbf{p}_{\mathrm{k}}, \mathbf{q}_{\mathrm{k}}, \mathbf{w}_{\mathrm{k}}$ are the vectors of the corresponding matrix $\mathbf{P}, \mathbf{Q}$ and $\mathbf{W}$. Once $\mathbf{P}, \mathbf{Q}$ and $\mathbf{W}$ are computed, the regression matrix in equation (1) can be determined by $\mathbf{C}=\mathbf{W Q}$.

The above computation corresponds to a learning process. For new image frames, the myocardial motion can then be predicted according to the intensity distribution at the chest and/or diaphragm by using the established regression matrix $\mathbf{C}$. In the current study, we used $40 \%$ of the image frames located at the end of the perfusion sequence for learning. Only the first five principal components of the covariance matrix are kept in extracting the regression matrix $\mathbf{C}$, since they cover around $90 \%$ or more of total covariance.

\subsection{Validation and in vivo Data Acquisition}

To validate the effectiveness of the proposed model extraction scheme for motion prediction, a simulated MR myocardial perfusion image sequence has been created. This data set is used to validate the registration process in a situation where the correct deformation is known. This also allows us to test the method in a variety of different conditions. The structure of the image was based on an example short axis slice of the heart. The images consisted of 6 anatomical features: myocardium of the left ventricle, myocardium of the right ventricle, blood pool of the left ventricle, blood 
pool of the right ventricle, the diaphragm, the chest wall. In addition, we introduced two distinct defects in the myocardium of the left ventricle these being a subendocardial defect and a transmural defect. This gave nine distinct regions that were spatially defined by a static bitmap image. The temporal intensity of these regions was defined by time series curves based on real values from example data sets. Subsequently, the image sequence was warped so as to simulate respiratory motion. This motion vector was based on a real motion vector from the inverse of the motion vector produced from the registration of a real sequence. This dataset was used to evaluate the proposed 2D free form registration, self-adaptive learning and prediction with PLSR. After numerical verification, nine in vivo data sets acquired from patients with known coronary artery disease were used to further validate the proposed technique. These images were obtained using a 1.5T Siemens Sonata scanner $(200 \mathrm{~T} / \mathrm{m} / \mathrm{s} ; 40$ $\mathrm{mT} / \mathrm{m}$ ) with a four-element phased-array receiver coil. The first-pass perfusion data was acquired with a FLASH sequence $(\mathrm{Tr}=3.7 \mathrm{~ms}$ ) that consists of three saturationrecovery short-axis slices per cardiac cycle, for 50 cycles during the first-pass of GdDTPA (cubital vein, $0.1 \mathrm{mmol} / \mathrm{kg}, 3 \mathrm{ml} / \mathrm{s}$ ) with a field of view of $400 \mathrm{~mm}$ (128 pixels) by $300 \mathrm{~mm}$ (64 pixels).

The accuracy of the deformation correction technique for the patient data sets was assessed by two independent observers by using an image analysis package (CMRtools, Imperial College). The scoring was done through the measurement of displacements in millimeters of predefined landmarks on the septal, anterior and posterior segments. The derived values were then averaged over the entire sequence, and between the three chosen locations.

\section{$3 \quad$ Results}

Figure 2 illustrates the internal correlation between respiration and myocardial deformation. For simplicity, a single input trace is given and patterns of respiration at 18 , $26,31,35,40$, and 46 cardiac cycles from the beginning of the acquisition are provided. Figure 3(a) demonstrates the accuracy of the free-form image registration technique and the modeling approach based on PLSR. The temporal trace of the input bolus is also provided as a reference. It is evident that the overall accuracy of the PLSR approach is significantly better than that of using the normal registration method. The reason for the registration technique to perform poorly at the beginning the sequence was due to the application of an inversion pulse to nullify signals from the myocardium before the arrival of the contrast bolus. Figure 3(b) shows the corresponding assessment results for the patient data sets, illustrating the overall improvement of the motion correction results where only $40 \%$ of the image frames are used for motion prediction.

\section{Discussion and Conclusions}

Image registration is a crucial step in quantifying myocardial perfusion images. The use of conventional image registration is useful in aligning inter frame object deformations. For first-pass MR myocardial perfusion imaging, a direct application of the method can be problematic, especially before/during the arrival of the contrast bolus. During this period, the signal from the myocardium is minimum, and thus leads to 
large registration errors. This effect has clearly been demonstrated in our simulation data as shown in Figure 3. PLSR is a method for motion prediction where both the input and the output response data contain significant redundancies. In our study, we have used the latter $40 \%$ of the total image sequence for learning, since these images have a relatively clear structure for almost all of the anatomy. The predicted deformation distribution has shown to be reliable. This not only reduces the number of frames requiring traditional registration down to $40 \%$, but also significantly improves the consistency of the derived deformation vectors. The study represents the first attempt of using PLSR for deformation modelling, which should have important value for other deformation prediction applications including motion adaptive radiotherapy and imaging, where the deformation of the target organ can be predicted from externally measurable signals.

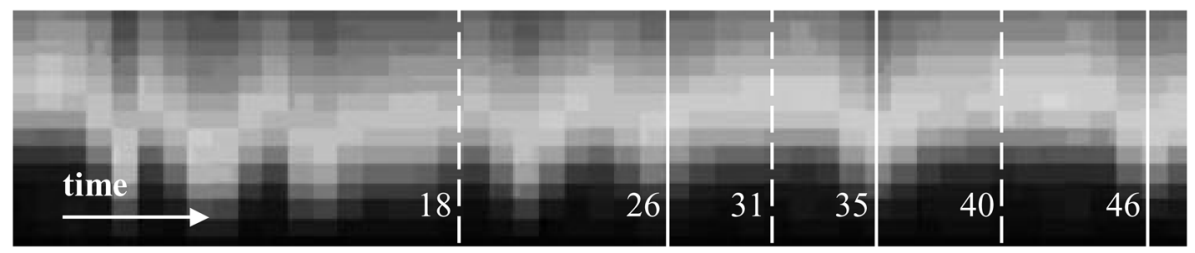

(a)
frame 18
frame 31
frame 40

frame 26

frame 35

frame 46

(b)

Fig. 2. An example showing the internal correlation between respiration and myocardial deformation. (a) A typical intensity distribution over time obtained from the chest wall and (b) the associated displacement vectors of the myocardium at six different time instants of the perfusion sequence 
(a) Residual motion after registration (synthetic data)

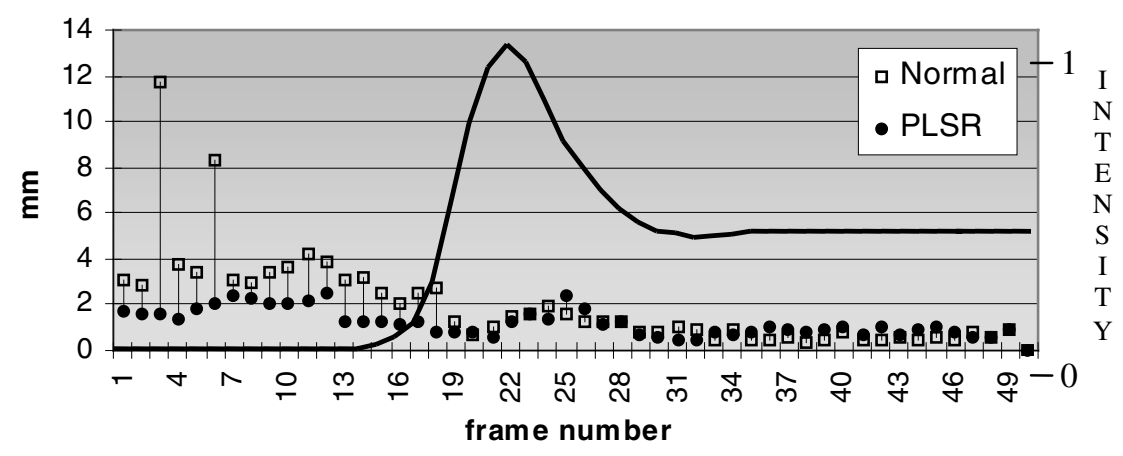

(b) Residual motion after registration (patient data)

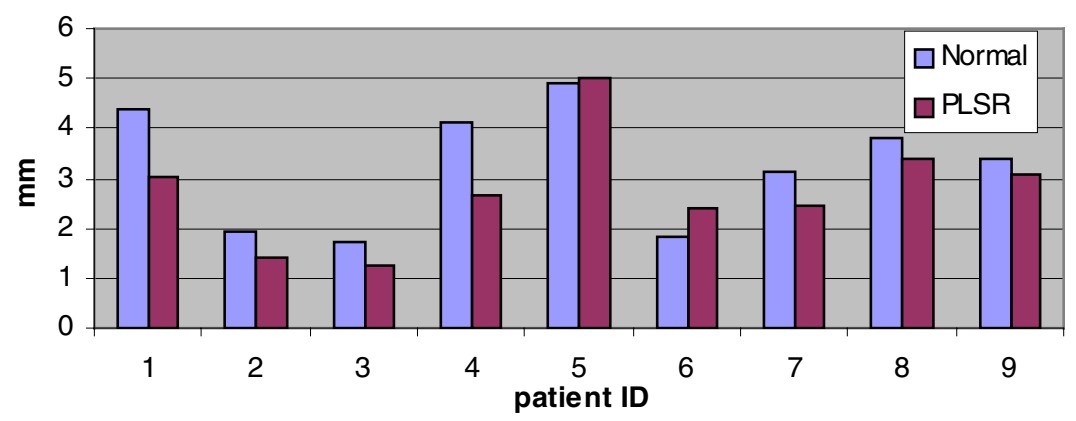

Fig. 3. Detailed error analysis for the proposed PLSR motion modeling approach with (a) synthetically generated perfusion data and (b) first-pass perfusion images from 9 patients with known coronary artery disease

\section{References}

1. Wilke N, Simm C, Zhang J, Ellermann J, Ya X, Merkle H, Path G, Ludemann H, Bache RJ, Ugurbil K. Contrast-enhanced first pass myocardial perfusion imaging: correlation between myocardial blood flow in dogs at rest and during hyperemia. Magn Reson Med. 1993; 29(4): 485-97.

2. Wilke N, Jerosch-Herold M, Stillman AE, Kroll K, Tsekos N, Merkle H, Parrish T, Hu X, Wang Y, Bassingthwaighte J, et al. Concepts of myocardial perfusion imaging in magnetic resonance imaging. Magn Reson Q. 1994; 10(4): 249-86.

3. Keijer JT, van Rossum AC, van Eenige MJ, Karreman AJ, Hofman MB, Valk J, Visser CA. Semiquantitation of regional myocardial blood flow in normal human subjects by first-pass magnetic resonance imaging. Am Heart J. 1995; 130:893-901.

4. Schwitter J, Debatin JF, von Schulthess GK, McKinnon GC. Normal myocardial perfusion assessed with multishot echo-planar imaging. Magn Reson Med. 1997; 37(1): 140-7.

5. Beache GM, Kulke SF, Kantor HL, Niemi P, Campbell TA, Chesler DA, Gewirtz H, Rosen BR, Brady TJ, Weisskoff RM. Imaging perfusion deficits in ischemic heart disease with susceptibility-enhanced T2-weighted MRI: preliminary human studies. Magn Reson Imaging. 1998; 16(1): 19-27. 
6. Wilke N, Kroll K, Merkle H, Wang Y, Ishibashi Y, Xu Y, Zhang J, Jerosch-Herold M, Muhler A, Stillman AE, et al. Regional myocardial blood volume and flow: first-pass MR imaging with polylysine-Gd-DTPA. J Magn Reson Imaging. 1995; 5(2): 227-37.

7. Larsson HBW, Stubgaard M, Søndergaard L, Henriksen O. In vivo quantification of the unidirectional influx constant for Gd-DTPA diffusion across the myocardial capillaries with MR imaging. J Magn Reson Imaging. 1994; 4: 433-40.

8. Cullen JH, Horsfield MA, Reek CR, Cherryman GR, Barnett DB, Samani NJ. A myocardial perfusion reserve index in humans using first-pass contrast-enhanced magnetic resonance imaging. J Am Coll Cardiol. 1999; 33: 1386-94.

9. Ablitt N, Gao JX, Gatehouse P, Yang GZ. Motion Decoupling and Registration for 3D Magnetic Resonance Myocardial Perfusion Imaging. International Conference on Computational Science. Amsterdam, The Netherlands, 2002.

10. Delzescaux T, Frouin F, De Cesare A, Philipp-Foliguet S, et al. Adaptive and selfevaluating registration method for myocardial perfusion assessment. Magn Reson Mater Phys Med. 2001; 13: 28-39.

11. Veeser S, Dunn MJ, Yang GZ. Multiresolution image registration for two-dimensional gel electrophoresis. Proteomics. 2001; 1: 856-870.

12. Wold, H. Soft modelling with latent variables: the nonlinear iterative partial least squares approach. Perspectives in probability and Statistics: Papers in honour of M.S. Barlett, (J. Gani, ed). London: Academic Press. 1975: 114-142.

13. Bhupinder S. Dayal, Jhon F. MacGregor. Recursive exponentially weighted PLS and its applications to adaptive control and prediction. J Proc Cont. 1997; 7: 169-179.

14. Blanco M, Coello J, Elaamrani M, Iturriaga H, Maspoch S. Partial least-squares regression for the quantitation of pharmaceutical dosages in control analyses. J Pharm. Biomed Anal. 1996; 15: 329-338. 